J. Phys. IV France 130 (2005) 141-151

(C) EDP Sciences, Les Ulis

DOI: $10.1051 /$ jp4:2005130010

\title{
La dynamique collective des membranes bicouches de modèle étudié par diffusion inélastique de neutrons
}

\author{
M.C. Rheinstädter ${ }^{1}$ et T. Salditt ${ }^{2}$ \\ ${ }^{1}$ Institut Laue-Langevin, 6 rue Jules Horowitz, BP. 156, 38042 Grenoble Cedex 9, France \\ e-mail : rheinstaedter@ill.fr \\ ${ }^{2}$ Institut für Röntgenphysik, Friedrich-Hund-Platz 1, 37037 Göttingen, Germany
}

\begin{abstract}
Résumé. Nous avons étudié la dynamique collective à courte longueur d'onde dans les bicouches du DMPC deutérié par diffusion inélastique de neutrons $[1,2]$. Dans cet exposé, nous examinerons la préparation expérimentale pour étudier la dynamique collective des membranes sur des instruments à trois axes, les résultats de la diffusion élastique et inélastique de neutrons et la forme particulière des relations de dispersion $\hbar \omega\left(\mathrm{Q}_{r}\right)$ dans la phase gel et fluide de ce système modèle. La dépendance en température des excitations inélastiques indique une coexistence de phase entre les domaines gel et fluide sur un large gamme de températures.
\end{abstract}

Table des matières

1 Introduction

2 Préparation des échantillons et de l'environnement

3 Diffusion inélastique de neutrons avec des spectromètres

à trois axes

4 Structure et dynamiques (collectives) des bicouches de lipides

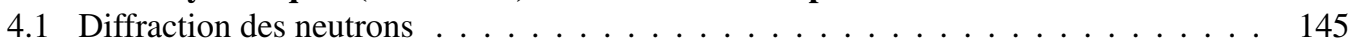

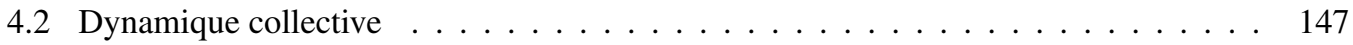

5 Conclusion

\section{INTRODUCTION}

Les membranes phospholipides sont étudiées de façon intensive en tant que systèmes modèles simples pour comprendre les aspects structurels et physiques fondamentaux de leurs équivalents biologiques beaucoup plus complexes [3]. La diffusion de neutrons peut contribuer à l'élucidation de la structure moléculaire, qui est bien documentée dans la littérature, ainsi qu'à la compréhen- sion de la dynamique moléculaire et supramoléculaire des bicouches de lipides. Les propriétés dynamiques sont souvent moins bien comprises dans les systèmes biomoléculaires, mais elles sont importantes pour de nombreuses propriétés fondamentales des biomatériaux, par exemple, les propriétés élastiques et les forces d'interaction. En outre, la dynamique des membranes de lipides sur de petites échelles de longueur moléculaire détermine ou affecte fortement les aspects fonctionnels, tels que la diffusion et le transport parallèle ou perpendiculaire à travers une bicouche. Dans cet exposé, nous examinerons l'utilisation de la diffusion inélastique de neutrons pour étudier les mouvements collectifs à faible distance des chaînes acyles des lipides.

Ainsi qu'ils sont représentés dans la Fig. 1 (a), les lipides sont composés d'une tête polaire hydrophile et de chaînes acyles hydrophobes. Dans une solution aqueuse, ils se rassemblent spontanément 


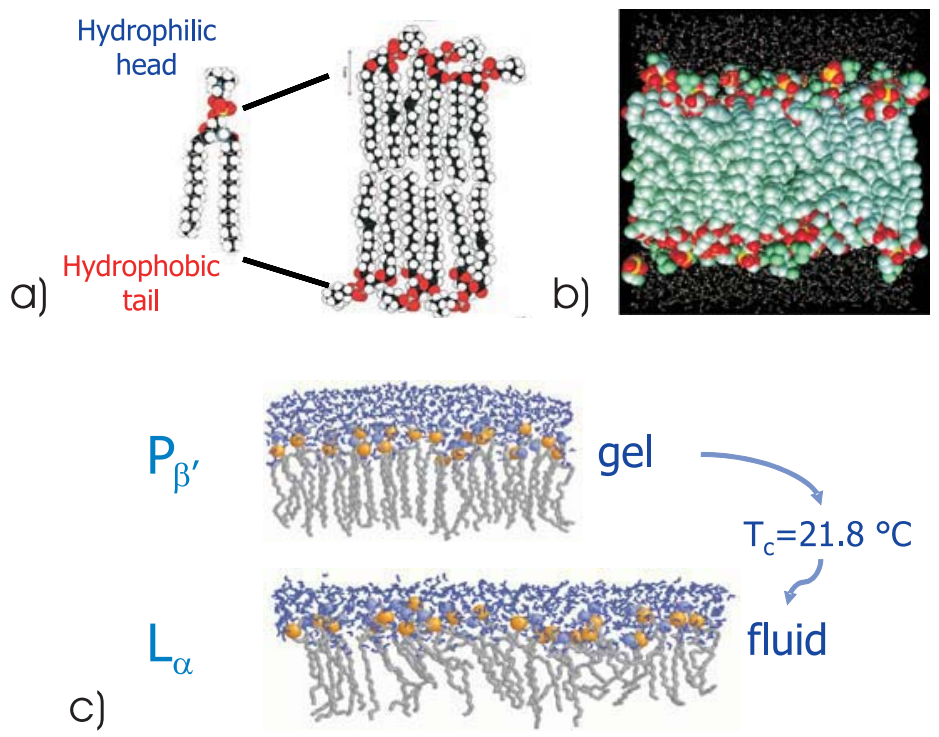

Figure 1. (a) Molécule de lipides avec une tête polaire hydrophile et des chaînes acyles hydrophobes. Dans une solution aqueuse, les lipides s'organisent spontanément en bicouches. (b) Modèle "réaliste" d'une membrane modèle à partir de simulations de Dynamiques Moléculaires (Heller et al. [4]). (c) "Transition principale" dans une bicouche du DMPC déutérié (-d54) à partir de la phase gel plus rigide ("phase ondulatoire"), $\mathrm{P}_{\beta^{\prime}}$ ) dans la phase fluide $\mathrm{L}_{\alpha}$.

en bicouches, les têtes polaires hydrophiles s'orientant ainsi vers l'eau. La Fig. 1 (b) présente une illustration plus "réaliste" de cette membrane modèle telle qu'elle est obtenue à partir de simulations de Dynamiques Moléculaires. Le système lipides/eau comprend une très grande variété de phases, en fonction de la température et du degré d'hydratation de la bicouche. Les phases correspondantes diffèrent, respectivement, en compacité des molécules de lipides et en orientation et mobilité des chaînes acyles des lipides. La "transition principale" est d'un grand intérêt dans la thermodynamique des bicouches de lipides, comme indiqué dans la Fig. 1 (c), qui passe d'une phase gel plus rigide ("phase ondulatoire", $\mathrm{P}_{\beta^{\prime}}$ ) à la phase fluide $\mathrm{L}_{\alpha}$. La transition du composé deutérié entièrement hydraté (DMPC $-\mathrm{d} 54$ ) que nous avons utilisé pour les expériences inélastiques de neutrons se produit à $\mathrm{T}=21,5^{\circ} \mathrm{C}$. Les bicouches de lipides que nous examinerons dans cet exposé sont composées d'un seul type de lipides, sans ajout de cholestérol ou de protéines, et peuvent servir de système modèle simple pour les membranes biologiques. L'étude des fluctuations dynamiques des membranes reste un défi expérimental important pour la biophysique actuelle, en particulier en ce qui concerne l'état biologique correspondant $L_{\alpha}$. Dans le cas de membranes simples, le signal de diffusion est de loin insuffisant pour une étude quantitative de la diffusion inélastique. Pour maximiser le signal de diffusion, nous avons préparé des échantillons multilamellaires composés d'empilements de plusieurs milliers de bicouches de lipides séparées par des couches d'eau, entraînant une structure de symétrie smectique A. L'ordre orientationnel élevé des échantillons qui donne lieu à des pics de Bragg et à des excitations intenses est une condition préalable à une analyse appropriée des fonctions de corrélation correspondantes. Il est très surprenant que la diffusion inélastique de neutrons ait été appliquée seulement très récemment à l'étude des membranes de lipides [1,2], fournissant une méthode unique pour étudier la dynamique collective (relations de dispersion) des macromolécules. Le caractère modèle du système permet d'étudier les excitations élémentaires dans les bicouches de lipides. Entre autres, il y a les modes locaux, qui sont des mouvements de molécules de lipides simples et les excitations collectives, c'est-à-dire, le mouvement cohérent de plusieurs molécules des membranes comme dans l'exemple présenté sur la Fig. 2. La diffusion rotationnelle et latérale, les vibrations et les rotations individuelles 
des molécules de lipides peuvent être étudiées par l'intermédiaire, par exemple, de la diffusion inélastique incohérente de neutrons, de la résonance magnétique nucléaire ou de la spectroscopie diélectrique. Par contre, seule la diffusion inélastique cohérente de neutrons ou la diffusion inélastique de rayons x peuvent permettre d'élucider les excitations collectives, par exemple, les fluctuations de densité à courte longueur d'onde ou les modes d'ondulation de la bicouche. Les mouvements moléculaires collectifs sont supposés jouer un rôle important dans les fonctions biologiques clés [6], en particulier les fluctuations de densité collectives à faible longueur d'onde, que nous examinerons ici, liées aux phénomènes de transport à l'intérieur et à travers la bicouche [7]. L'échelle de longueur de ces fluctuations est d'environ $20 \AA$ (environ trois molécules de lipides), comme indiqué dans la Fig. 2 (b).

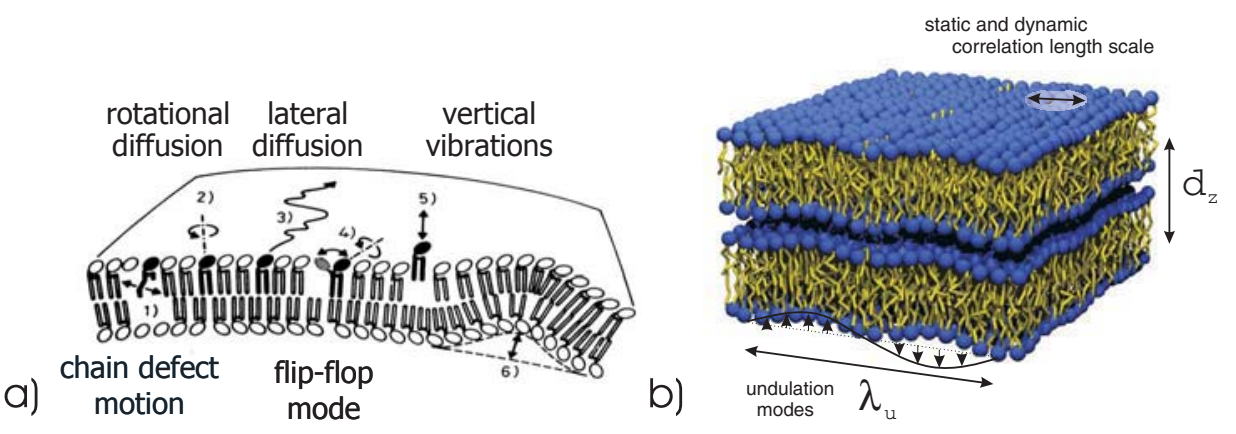

Figure 2. Certains modes dynamiques élémentaires dans les bicouches de lipides. (a) Les modes locaux incluent une diffusion et des mouvements individuels des molécules de lipides (voir Pfeiffer et al. [5] pour une description détaillée des différents mouvements). (b) Les excitations collectives sont des mouvements cohérents de plusieurs molécules des membranes, comme par exemple,, l'ondulation à grande longueur d'onde et les modes de flexion des membranes.

L'exposé est organisé comme suit: après cette introduction, nous examinerons la préparation des échantillons et l'environnement des échantillons pour les phases de lipides multilamellaires sur support solide. La section 3 présente les récents progrès réalisés dans l'application de la diffusion inélastique de neutrons classique à l'aide de spectromètres à trois axes pour l'étude la dynamique à faible distance des bicouches de lipides, comme le mouvement collectif des chaînes acyles. Nous examinerons les résultats relatifs à deux aspects majeurs: nous examinerons de façon détaillée les excitations collectives et la forme particulière des relations de dispersion trouvées dans une bicouche du DMPC $-\mathrm{d} 54$. Nos mesures visent à établir une coexistence de phase entre les domaines gel et fluide sur un large spectre de températures. L'article se termine par un petit résumé.

\section{PRÉPARATION DES ÉCHANTILLONS ET DE L'ENVIRONNEMENT}

Une réalisation importante à été la préparation des échantillons appropriée aux expériences de diffusion inélastique de neutrons avec un maximum d'échantillons de matériaux pour rendre les petits signaux inélastiques accessibles. Nous avons préparé des empilements de membranes fortement orientées en étalant une solution de lipides typiquement de $25 \mathrm{mg} / \mathrm{ml}$ dans du trichloréthylène/chloroforme $(1: 1)$ sur des plaquettes de 2 pouces, suivi d'un séchage sous vide et d'une hydratation par vapeur d'eau lourde $\left(\mathrm{D}_{2} \mathrm{O}\right)$ [8], voir le croquis de la Fig. 3 (a). Le nombre d'empilements multilamellaires par plaquette peut être estimé à plusieurs milliers, avec une épaisseur totale d'environ $15 \mu \mathrm{m}$. La cristallisation des lipides, qui détruit ensuite la structure en couches, établit une limite naturelle du nombre maximum de couches de lipides par plaquette. Jusqu'à quatorze de ces plaquettes séparées par de petites couches d'air ont été combinées et alignées les unes par rapport aux autres pour créer un "échantillon sandwich" composé de plusieurs milliers de bicouches de lipides fortement orientées, avec une masse totale de plus de $300 \mathrm{mg}$ 


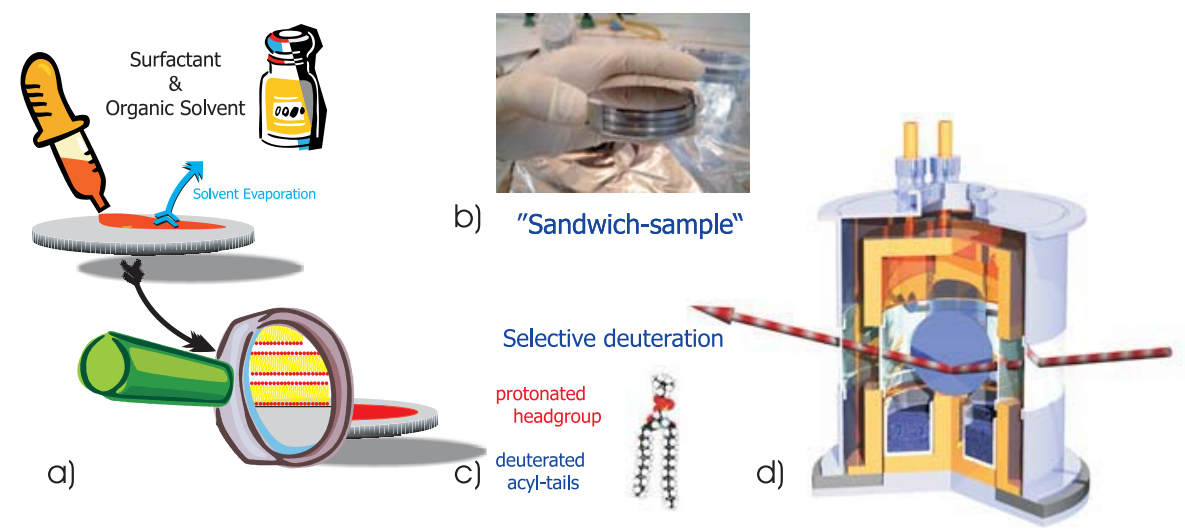

Figure 3. (a) Croquis de la préparation des échantillons. (b) Photographie de "l'échantillon sandwich" préparé pour les expériences de diffusion inélastique de neutrons. (c) Par deutération sélective, le poids des mouvements collectifs des chaînes acyles de lipide est augmenté par rapport aux autres contributions à la section efficace de diffusion inélastique. (d) Schéma de la chambre utilisée pour les expériences sur les neutrons pour contrôler la température et l'humidité des bicouches.

de DMPC déutérié. La figure 3 (b) présente une photographie de l'échantillon pour les neutrons. La mosaicité de l'échantillon (répartition des vecteurs normaux), c'est-à-dire l'alignement des membranes par rapport au substrat et l'alignement des substrats les uns par rapport aux autres, était meilleure que $0,6^{\circ}$. Pour contrôler la température et l'hydratation pendant l'expérience, les échantillons étaient conservés dans une chambre ferm'ee en aluminium à température et humidité contrôlée (Fig. 3 (d)). L'hydratation des membranes de lipides était réalisée à l'aide de deux bains chauffants contrôlés, reliés à l'échantillon et à un réservoir d'eau lourde pour permettre l'hydratation de l'échantillon à partir de la phase vapeur du $\mathrm{D}_{2} \mathrm{O}$. Le spectromètre à trois axes a été ensuite utilisé pour mesurer, en une seule opération, le facteur de structure statique $\mathrm{S}\left(\mathrm{Q}_{r}\right)$ et dynamique $\mathrm{S}\left(\mathrm{Q}_{r}, \omega\right)$ dans le plan des membranes. Par deutération sélective des chaînes acyles (Fig. 3 (c)), le poids des mouvements collectifs est fortement augmenté par rapport aux autres contributions à la section efficace de diffusion inélastique.

\section{DIFFUSION INÉLASTIQUE DE NEUTRONS AVEC DES SPECTROMÈTRES À TROIS AXES}

Le spectromètre à trois axes a été très efficace dans l'étude des excitations collectives de la physique de la matière condensée, c'est-à-dire, des phonons et des magnons dans les cristaux, mais il n'a jusqu'à présent pas été appliqué aux membranes de lipides. Les avantages des spectromètres à trois axes sont leur conception et leur fonctionnement relativement simples et l'utilisation efficace du flux de neutrons incidents pour sonder certains points dans l'espace réciproque $(\mathrm{Q}, \omega)$. La figure 4 (a) présente un schéma d'un spectromètre à trois axes. En faisant varier les trois axes de l'instrument, l'axe de rotation du monochromateur, de l'échantillon et de l'analyseur, les vecteurs d'onde $\mathrm{k}_{i}$ et $\mathrm{k}_{f}$ et les énergies $\mathrm{E}_{i}$ et $\mathrm{E}_{f}$ des neutrons, respectivement, incidents et diffusés peuvent être déterminés. $\mathbf{Q}$, le transfert d'impulsion à l'échantillon, et le transfert d'énergie, $\omega$, sont ensuite définis par les lois de conservation de l'impulsion et de l'énergie: $\mathbf{Q}=\mathbf{k}_{\mathbf{f}}-\mathbf{k}_{\mathbf{i}}$ et $\omega=E_{i}-E_{f}$. La gamme accessible $(\mathbf{Q}, \omega)$ sur IN12 pour une énergie fixe des neutrons diffusés $\mathrm{E}_{f}$ de $10 \mathrm{meV}$ couvre bien le spectre des excitations Q- $\omega$ sondée ( Fig. 4 (b)). Il est juste limité par le spectre en 'energie de neutrons incidents offert par le guide de neutrons ainsi que par les restrictions dues à l'encombrement mécaniques du spectromètre. La résolution en énergie de l'instrument est $\Delta=500 \mu \mathrm{eV}$ dans cette configuration. En choisissant des énergies de neutrons incidents et des transferts d'énergie plus petits, la résolution en énergie peut être améliorée. Les mesures ont été 

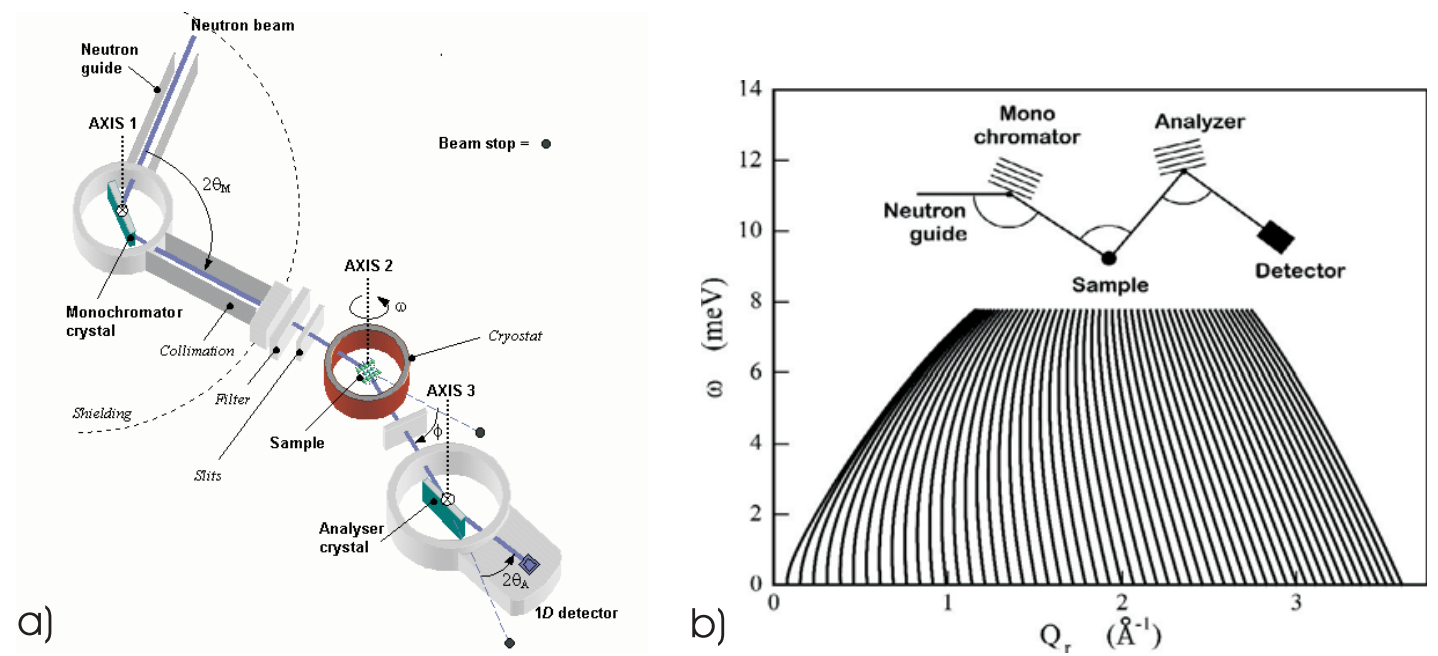

Figure 4. (a) Schéma d'un spectromètre à trois axes [9] et (b) spectre Q- $\omega$ - de IN12 accessible pour une énergie finale des neutrons diffusés de $\mathrm{E}_{f}=10 \mathrm{meV}$.

effectuées avec les spectromètres à trois axes IN12 sur source froide et IN3 sur source thermique au réacteur à haut flux de l'ILL à Grenoble, France. La combinaison des neutrons froids et thermiques rend accessible le spectre d'excitations sur une gamme allant de 0,5 à $30 \mathrm{meV}$. L'utilisation d'empilements de membranes fortement orientées a permis d'aligner parfaitement le vecteur de diffusion $\mathbf{Q}$ par rapport aux bicouches de lipides. $\mathbf{Q}$ peut être placé dans le plan des membranes pour mesurer les corrélations statiques, $\mathrm{S}\left(\mathrm{Q}_{r}\right)$, ou dynamique $\mathrm{S}\left(\mathrm{Q}_{r}, \omega\right)$ dans le plan. En faisant tourner l'échantillon de $90^{\circ}$, $\mathbf{Q}$ peut être placé perpendiculaire aux bicouches pour examiner les corrélations interlamellaires afin de déterminer l'espace interlamellaire et l'épaisseur de la couche d'eau. Par conséquent, le spectromètre à trois axes offre la possibilité de mesurer la réflectivité et la dynamique dans le plan avec le même instrument sans changer de configuration. Cela constitue un avantage inestimable étant donné que l'état thermodynamique de la bicouche de lipides dépend non seulement de la température et de l'humidité relative, mais également du taux de refroidissement ou d'échauffement de la préparation et de l'historique thermique de l'échantillon.

\section{STRUCTURE ET DYNAMIQUES (COLLECTIVES) DES BICOUCHES DE LIPIDES}

\subsection{Diffraction des neutrons}

La figure 5 présente un schéma de l'espace réciproque dans la phase gel de la bicouche de DMPC deutériée à $\mathrm{T}=20^{\circ} \mathrm{C}$. Avec le vecteur de diffusion $\mathbf{Q}$ placé dans le plan des membranes, les corrélations statiques dans le plan peuvent être mesurées. A $\mathrm{Q}_{r}=1.5 \AA^{-1}$ il y a le pic de corrélation de la châne inter acyle. En raison de la dimension réduite du système, l'intensité est étalée dans le sens perpendiculaire à la direction $z$. La réflectivité est mesurée avec $\mathbf{Q}$ perpendiculaire aux membranes et présente des pics de Bragg bien développés. La résolution en $\mathrm{Q}$ de la réflectivité mesurée est assez basse comparée aux résultats des réflectomètres dédiés mais les pics de Bragg peuvent manifestement être analysés et donner des informations précieuses pour caractériser la structure en couches. La qualité des données de diffraction par rapport au rapport de l'intensité de pic sur bruit de fond est très élevée car l'analyse en énergie supprime le bruit de fond provenant par exemple, de l'hydrogène et améliore radicalement le rapport signal sur bruit.

La figure 6 (a) présente des courbes de réflectivité, ainsi que les résultats du profil de densité obtenus par le biais d'une approche par synthèse de Fourier dans la Fig. 6 (b). Les données ont été enregistrées 


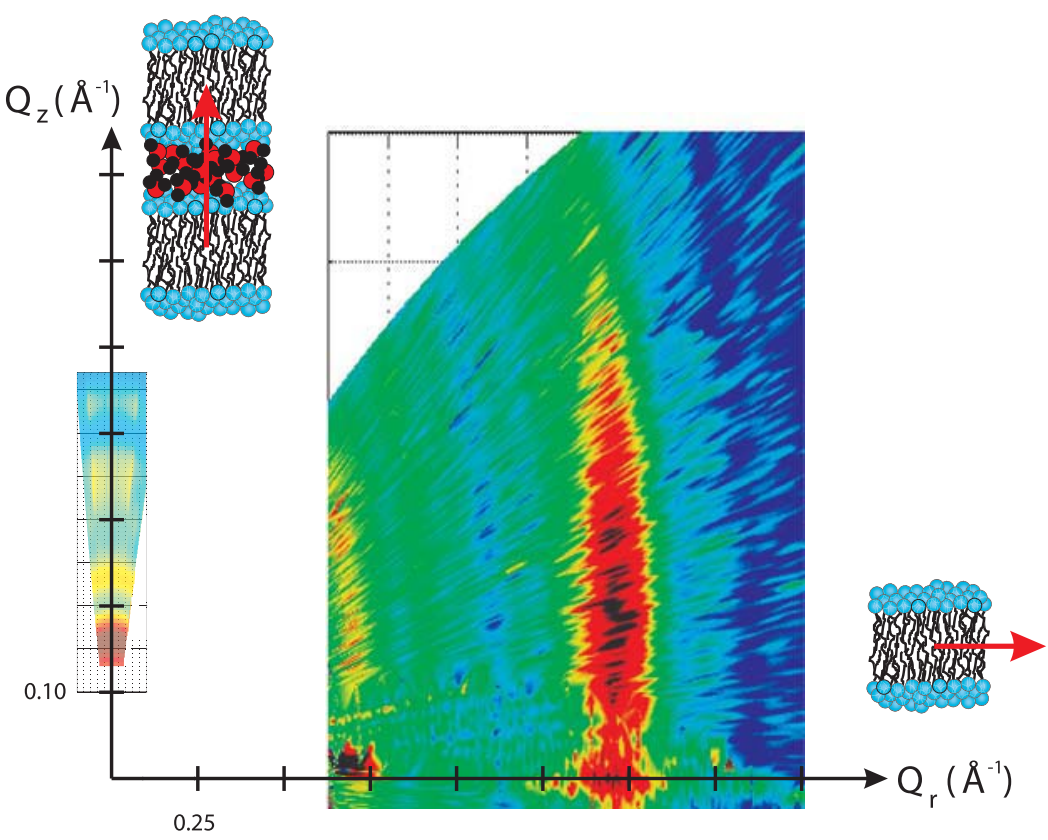

Figure 5. Schéma de l'espace réciproque dans les bicouches de lipides. Les pics de réflectivité de Bragg sont mesurés avec $\mathbf{Q}$ placé perpendiculaire aux membranes $\left(\mathrm{Q}_{z}\right)$. Avec $\mathbf{Q}$ dans le plan des membranes, il y a le pic de corrélation de la chaîne inter acyle à $\mathrm{Q}_{r}=1.5 \AA^{-1}$.
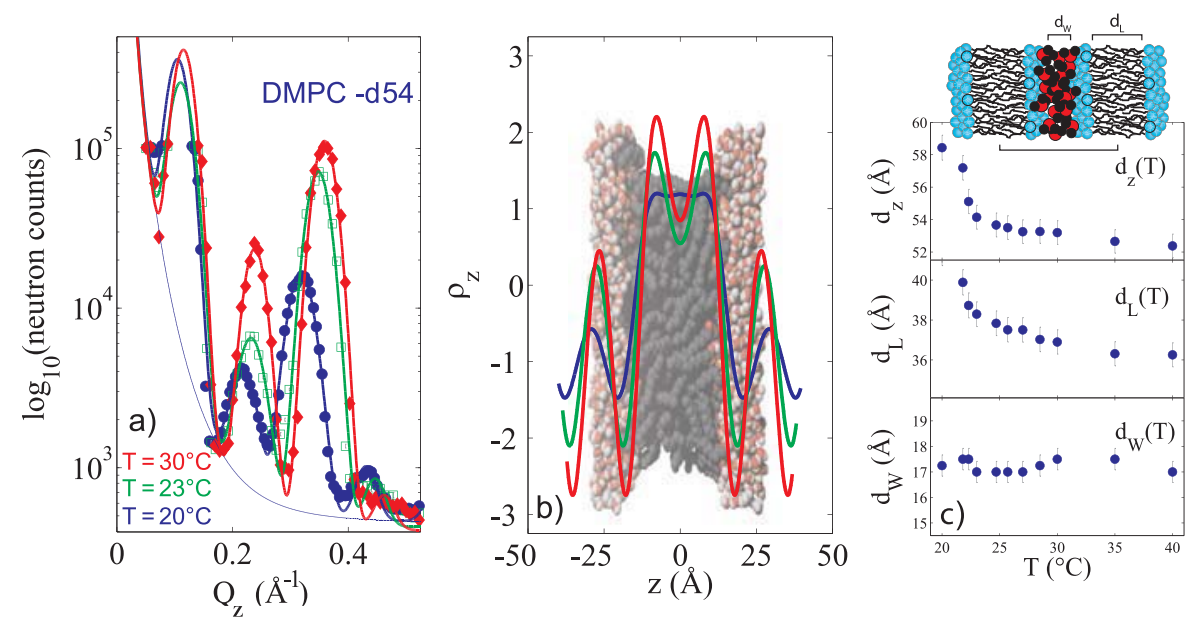

Figure 6. (a) Courbes de réflectivité et (b) profils de densité de longueur de diffusion obtenus à partir de la synthèse de Fourier sur les intensités de pic intégrées. (c) Dépendance à la température de la périodicité $d_{z}$, de la couche d'eau $d_{w}$ et de la couche hydrophobe $d_{L}$ (chaînes acyles deutériées), de l'épaisseur du DMPC à proximité de la transition de la phase principale. Les valeurs sont évaluées à partir des profils de densité comme indiqué dans la Fig. 6 (b) et présentent une dilatation anormale.

comme une fonction de $\mathrm{T}$ au dessous et au dessus de la transition de phase principale simultanément avec les données inélastiques examinées plus loin. Les paramètres de structure du profil de la bicouche en fonction de la température sont présentés dans la Fig. 6 (c). Les deux différentes phases présentent des différences distinctes dans $\rho_{z}$ : tandis que la densité de longueur de diffusion dans le centre de la bicouche 

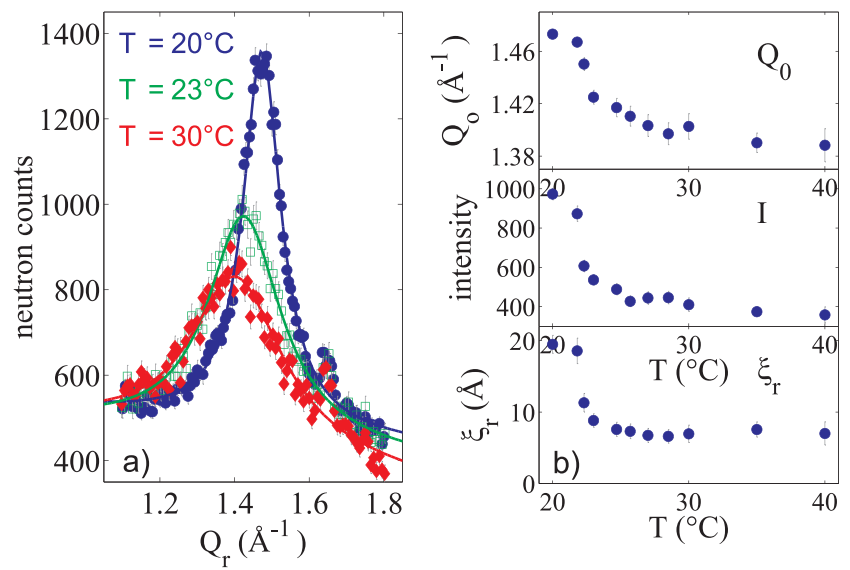

Figure 7. (a) Scans en $\mathrm{Q}$ du pic de la chaîne acyle pour les températures $\mathrm{T}=20,23$ et $30^{\circ} \mathrm{C}$ à travers la transition de phase principale de la bicouche du DMPC -d54. (b) $\mathrm{Q}_{0}$, intensité et longueur de corrélation $\xi_{r}$ extraites de la position du pic, de l'amplitude et de la largeur pour toutes les températures mesurées entre $\mathrm{T}=20$ et $40^{\circ} \mathrm{C}$.

dans la phase gel $\left(\mathrm{T}=20^{\circ} \mathrm{C}\right)$ est presque de forme parallélépipédique, elle perd sa forme dans la phase fluide dynamique et moins compacte à $\mathrm{T}=30^{\circ} \mathrm{C}$. Nous avons réalisé des scans en $\mathrm{Q}$ dépendants de la température à travers le pic de la chaîne inter acyle dans la gamme de températures autour de la transition de phase principale de $\mathrm{T}=20^{\circ} \mathrm{C}$ à $\mathrm{T}=40^{\circ} \mathrm{C}$ pour étudier les corrélations statiques dans le plan de la bicouche. La figure 7 (a) présente des exemples de scans en $\mathrm{Q}$ à $\mathrm{T}=20,23$ et $30^{\circ} \mathrm{C}$. Lorsqu'elle passe de la phase gel à la phase fluide, la position du pic passe à des valeurs de $\mathrm{Q}$ plus petites $\mathrm{Q}_{r}$ (distances moyennes entre proches voisins plus grandes) et les pics s'élargissent, indiquant une longueur de corrélation décroissante $\xi_{r}$ dans le plan des membranes. La figure 7 (b) donne $\mathrm{Q}_{0}$, l'amplitude et la longueur de corrélation $\xi_{r}$ telles qu'obtenues à partir de la position du pic, de l'amplitude et de la largeur pour toutes les températures mesurées. Bien que la transition de phase soit du premier ordre [10-12], les valeurs s'orientent vers un comportement critique (dilatation anormal) de la bicouche.

\subsection{Dynamique collective}

Un scan en énergie caractéristique du DMPC -d54 est présenté dans la Fig. 8 (a). Les données ont été recueillies à $\mathrm{T}=20^{\circ} \mathrm{C}$, dans la phase gel de la bicouche à $\mathrm{Q}=1,0 \AA^{-1}$. La cartouche présente un agrandissement sur les excitations de la bicouche dans la phase gel et fluide $\left(a ̀ \mathrm{~T}=30^{\circ} \mathrm{C}\right.$, neuf degrés au-dessus de la température de transition de la phase). Nous trouvons des pics bien prononcés dont la position et la largeur peuvent facilement être déterminées. Les scans inélastiques peuvent être analysés par la théorie généralisée à trois modes propres effectifs [13-15]. La fonction suivante a été utilisée pour un ajustement des moindres carrés:

$$
\begin{aligned}
\frac{S(Q, \omega)}{S(Q)}= & \frac{1}{\pi}\left(A_{0} \frac{\Gamma_{h}}{\omega^{2}+\Gamma_{h}^{2}}\right. \\
& \left.+A_{s}\left[\frac{\gamma_{s}+b\left(\omega+\omega_{s}\right)}{\left(\omega+\omega_{s}\right)^{2}+\gamma_{s}^{2}}+\frac{\gamma_{s}-b\left(\omega-\omega_{s}\right)}{\left(\omega-\omega_{s}\right)^{2}+\gamma_{s}^{2}}\right]\right)
\end{aligned}
$$

Le modèle se compose d'un mode central de diffusion de la chaleur, centré à $\omega=0 \mathrm{meV}$ (Lorentzian avec une largeur $\Gamma_{h}$ ), et de deux modes accoustiques, représentés par des lorentziennes à $\omega= \pm \omega_{s}$ et par un amortissement $\gamma_{s}[13,15]$. A partir de la largeur du mode central, et de la largeur et de la position des lignes Brillouin, la diffusivité thermique, la fréquence et l'amortissement de l'onde sonore peuvent être déterminées dans le cadre d'une théorie hydrodynamique. Pour ajuster les données des neutrons, nous 
devons ajouter une composante lorentzienne supplémentaire pour décrire la contribution quasi-élastique large associée aux degrés de liberté intramoléculaire et à la diffusion incohérente qui n'est pas vue par diffusion inélastique de rayons X. [14]. La figure 8 (b) présente un scan en énergie de la phase gel à $\mathrm{Q}_{r}=3,0 \AA^{-1}$ jusqu'à un transfert d'énergie de $30 \mathrm{meV}$. A l'exception de l'excitation dispersive due à des ondes de densité dans le plan, un deuxième mode (optique) non dispersif est observé à environ $\omega=14 \mathrm{meV}$ avec une largeur (FWHM) d'environ $13 \mathrm{meV}$, correspondant exactement aux prédictions de Tarek et al. [16] qui a attribué ce mode aux extrémités méthylées des chaînes acyles.
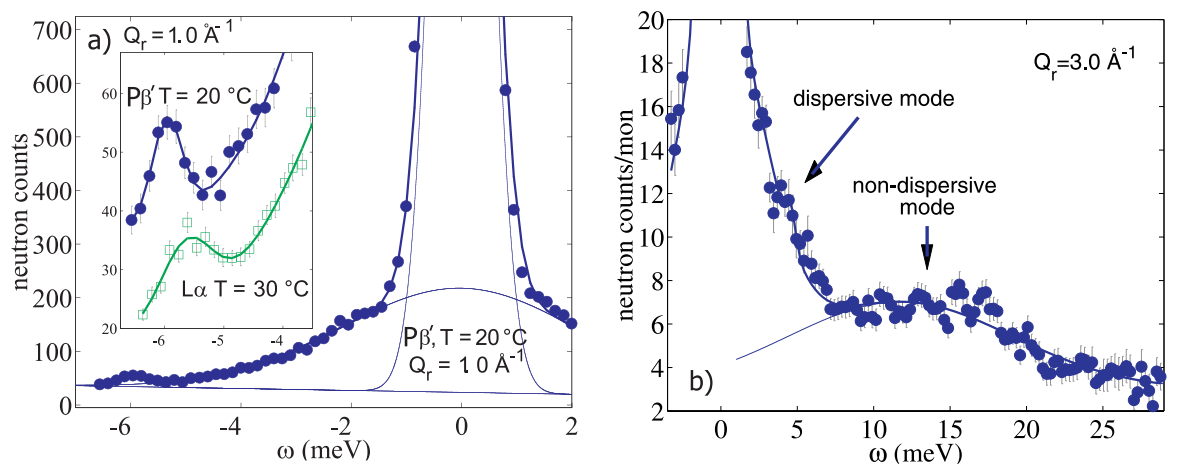

Figure 8. (a) Scan en énergie à une valeur $\mathrm{Q}_{r}$ de $1,0 \AA^{-1}$ dans la phase gel de la bicouche $\left(\mathrm{T}=20^{\circ} \mathrm{C}\right)$. La cartouche présente une vue agrandie des excitations de la bicouche dans la phase gel et la phase fluide (à $\left.\mathrm{T}=30^{\circ} \mathrm{C}\right)$. (b) scan à travers une gamme de transfert en énergie plus élevé $\left(\mathrm{Q}_{r}=3,0 \AA^{-1}\right.$, données IN3) présentant l'excitation dispersive et l'excitation non dispersive (optique) qui a été prédite par l'intermédiaire du calcul de dynamiqu moléculaire.
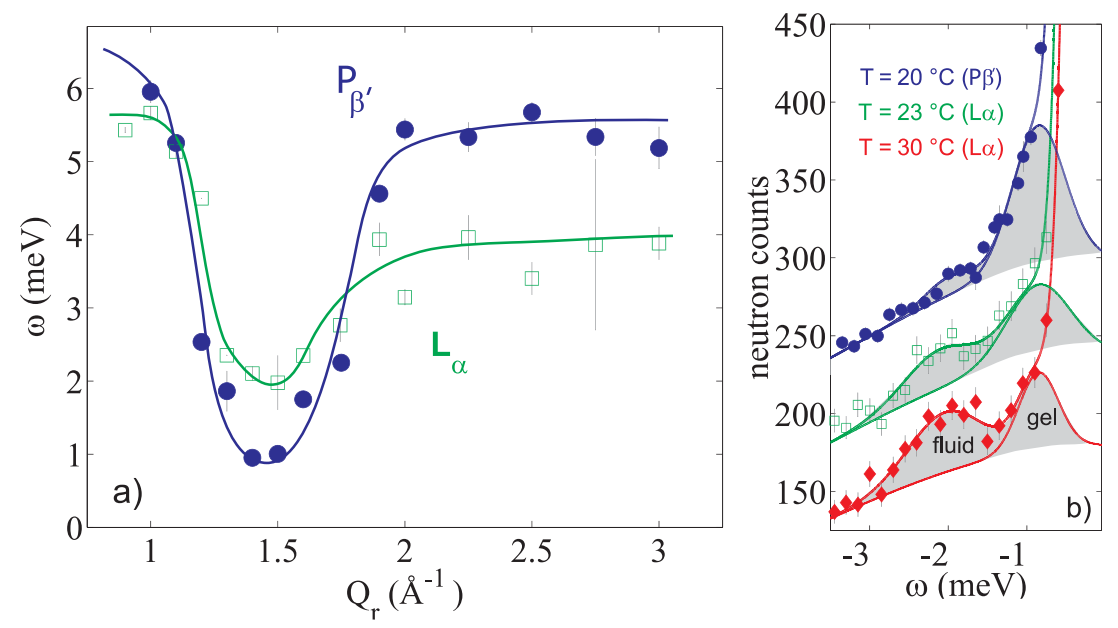

Figure 9. (a) Relations de dispersion dans la phase gel et la phase fluide de la bicouche du DMPC mesurées par plusieurs scans à $\mathrm{Q}$ constants à des valeurs $\mathrm{Q}$ allant de $\mathrm{Q}=0,7$ à $3,0 \AA^{-1}$. (b) Scans en énergies au minimum de la dispersion à $\mathrm{Q}=1,5 \AA^{-1}$ pour des températures $\mathrm{T}=20,23$ et $30^{\circ} \mathrm{C}$.

La figure 9 (a) présente la relation de dispersion dans la phase gel et la phase fluide ainsi qu'elle a été mesurée par plusieurs scans à $Q$ constants à des valeurs $Q$ allant de $Q=0,7 \AA^{-1}$ à $Q=3,0 \AA^{-1}$. La dispersion du fluide a été mesurée plus loin dans la phase fluide de la bicouche du DMPC. La relation de dispersion trouvée est similaire à celles des liquides parfaits, comme par exemple, l'argon liquide [17, 18], le néon liquide [19] ou l'hélium liquide [20]. Il semble que l'intérieur de la bicouche (les atomes C ou 
les groupes C-D des chaînes acyles des lipides) se comporte comme un quasi-liquide. Contrairement aux liquides parfaits, les atomes des molécules de lipides sont chimiquement liés les uns aux autres, ce qui entraîne une moindre mobilité et une diffusion plus faible. Contrairement aux liquides parfaits, où les excitations au-delà du premier maximum dans la relation de dispersion sont généralement déjà très suramortis et par conséquent difficiles à mesurer, les modes à des valeurs Q plus élevées dans les bicouches des lipides sont toujours relativement nets et donnent des satellites prononcés.

La forme particulière de la relation de dispersion peut être expliquée d'un point de vue qualitatif. Le scénario de base est le suivant: à un $\mathrm{Q}_{r}$ faible, des ondes sonores longitudinales dans le plan de la bicouche sont mesurées et donnent lieu à une augmentation linéaire de $\omega \propto \mathrm{Q}_{r}$, saturant à certaines valeurs maximales ("maxon"), avant qu'un $\Omega_{0}$ minimum prononcé ("roton") soit observé à $\mathrm{Q}_{0} \simeq 1,5 \AA^{-1}$, le premier maximum dans le facteur de structure statique $S\left(Q_{r}\right)$ (le pic de corrélation de la chaîne inter acyle). Du point de vue qualitatif, ceci peut être compris si $\mathrm{Q}_{0}$ est interprété comme étant la quasi-zone de Brillouin d'un liquide bidimensionnel. Les modes collectifs ayant une longueur d'onde de l'ordre de la distance moyenne entre proches voisins $2 \pi / \mathrm{Q}_{0}$ sont énergétiquement favorables et conduisent au minimum. Dans des cristaux parfaitement ordonnés, l'énergie des branches de phonons acoustiques descend à zéro au centre de zone. Le désordre statique et dynamique dans les bicouches de lipides conduit finalement à un minimum à énergie finie ("mode mou”). À des valeurs $\mathrm{Q}_{r}$ bien au-dessus du minimum, la relation de dispersion est dominée par un comportement de particules individuelles. Une théorie quantitative qui prédit les valeurs des énergies absolues du "maxon" et du "roton" sur la base de paramètres moléculaires manque jusqu'à présent. Cependant, la relation de dispersion, extraite des simulations de Dynamiques Moléculaires (DM) par une transformation de Fourier temporelle et spatiale des coordonnées moléculaires de l'espace réel [16], montre un accord excellent avec les données. La valeur de l'énergie du "maxon" et la branche de dispersions à grand Q sont plus élevés dans la phase gel que dans la phase fluide, la valeur de l'énergie du minimum de dispersion est en fait plus petite, suggérant un couplage plus fort entre les chaînes de lipides dans la configuration tout-trans de la phase gel. La gamme des petits $\mathrm{Q}$ est difficile à atteindre par la diffusion inélastique de neutrons en raison de la restriction cinétique, c'est-à-dire la relation de dispersion du neutron lui-même. Cette restriction n'existe pas pour la diffusion inélastique de rayons X; la "dispersion" d'un photon est une ligne droite avec une pente extrêmement raide. Mais en raison de la géométrie de rétrodiffusion et des angles de diffusion très petits, il est très difficile de séparer les rayons X diffusés à partir du faisceau direct dans l'expérience sur les rayons $\mathrm{X}$.

Etant donné qu'il s'agit d'un point très important de la relation de dispersion, nous avons effectué des mesures inélastiques en fonction de la température au niveau du minimum de la branche de dispersion entre $\mathrm{T}=20$ and $40^{\circ} \mathrm{C}$ avec une résolution en énergie améliorée. La figure 9 (b) présente les scans en énergie correspondants à $\mathrm{Q}=1,5 \AA^{-1}$. Dans la phase gel, nous trouvons une excitation à $\omega_{s}=-1 \mathrm{meV}$. A des températures plus élevées, les excitations du gel diminuent et une nouvelle excitation à $\omega_{s}=-2 \mathrm{meV}$ se développe. Nous associons la deuxième excitation à la phase fluide. L'affectation des excitations aux phases particulières se justifie par leur dépendance en température: dans chaque phase, il y a une excitation dominante. Les deux modes sont nettement dispersifs et changent d'énergie lorsqu'ils se déplacent à l'extérieur du minimum. Nous trouvons déjà des traces de "l'excitation du fluide" dans la phase gel. À $\mathrm{T}=30^{\circ} \mathrm{C}$, plus loin dans la phase fluide, il y a toujours "l'excitation du gel", indiquant une coexistence des domaines gel et fluide. Au même moment, les scans élastiques ne présentent pas de coexistence des deux phases, mais un comportement caractéristique bien connu: alors que la transition est de premier ordre, une dilatation pseudo-critique, c'est-à-dire, un changement continu de la distance interlamellaire dans la gamme de température autour $\mathrm{de}_{c}$, est observé pour le DMPC et les autres lipides [10, 11, 21]. Les changements de $d_{z}$ dans la Fig. 6 (c) sont accompagnés des changements correspondants dans la distance moyenne entre les chaînes dans la Fig. 7 (b). Pour ces deux paramètres, les données de diffraction montrent un changement continu, tandis que l'interprétation des courbes inélastiques indique une coexistence des domaines gel et fluide, en particulier bien au-dessus de la transition vers la phase fluide. Un point essentiel est la taille des domaines. La coexistence de domaines macroscopiques ayant des tailles supérieures à la 
longueur de cohérence $\xi_{n}$ des neutrons dans l'échantillon entraînerait une division du pic de la chaîne car les faisceaux diffusés des différents domaines ne peuvent plus interférer et à la place, les valeurs absolues des amplitudes diffusées devraient être sommées. Par conséquent, les tailles des domaines doivent être plus petites que $\xi_{n}$, qui peut être estimé à quelques centaines d' angström. Pour les domaines ayant des tailles inférieures à $\xi_{n}$, les ondes diffusées élastiquement devraient interférer et les données devraient présenter des valeurs moyennes $\mathrm{Q}_{0}$ dans les deux domaines. Les ondes inélastiquement diffusées ne peuvent pas interférer à cause de la différence de longueur d'ondes et les spectres devraient présenter deux excitations, ainsi qu'il est démontré dans l'expérience. Dans les systèmes cristallins, il est bien connu qu'un cristal subissant une transition de phase peut présenter des caractéristiques supplémentaires pour s'adapter aux contraintes macroscopiques, par exemple, pour empêcher une rupture du cristal due à une différence de volume des deux phases et aux grandes déformations correspondantes. De la même façon, la bicouche de lipides peut tenter de s'adapter à la différence de volume des phases gel et fluide lorsqu'elle passe à travers la transition principale. Une répartition des déformations correspondant à la formation de microdomaines peut alors empêcher une rupture du film de lipides. En raison de la mobilité des molécules de lipides, la formation, la taille et même le type de domaines constituent sans doute un processus très dynamique, au moins très proche de la température de transition dans lequel les deux types de domaines ont une probabilité égale. Nos résultats indiquent que même en profondeur dans les phase gel ou fluide respectivement, il y a des traces de domaines de fluide ou de gel. Cette coexistence des domaines de gel et de fluide pourrait constituer une vraie propriété de la bicouche de lipides et pourrait même se produire dans des bicouches "libres" sans aucunes contraintes externes de la surface ou du substrat.

\section{CONCLUSION}

Les mesures de la dynamique collective à courte longueur d'onde dans les bicouches de lipides constituent un domaine nouveau mais prometteur, car la dynamique collective joue probablement un rôle crucial dans les différentes fonctions biologiques. L'utilisation de spectromètres à trois axes permet de mesurer la structure et la dynamique, c'est-à-dire, la réflectivité, le pic de corrélation de la chaîne de lipides acylés et la dynamique dans le plan des membranes modèle sur une échelle de longueur moléculaire sans changer de conditions expérimentales. Nous avons mesuré la relation de dispersion dans la phase gel et la phase fluide du système modèle de DMPC - d54. L'interprétation des expériences dépendantes de la température s'oriente vers une nouvelle interprétation de la transition de phase gel-fluide et des excitations collectives dans les bicouches de lipides. D'autres études inélastiques aborderont l'influence des différentes têtes polaires et queues hydrophobes sur la dynamique collective. En outre, l'influence du cholestérol et des protéines actives des membranes sera, nous l'espérons, étudiée pour donner un nouvel aperçu de la fonctionnalité de ces systèmes. L'étude de systèmes de plus en plus complexes pourrait aboutir à une meilleure compréhension des membranes biologiques réelles.

Nous remercions toutes les personnes qui nous ont soutenu: Christoph Ollinger pour la préparation des échantillons et pour les bons moments passés à faire les expériences ensemble, Giovanna Fragneto pour son aide relative aux l'environnements échantillon et pour nos discussions fructueuses, Wolfgang Schmidt pour son soutien sur IN12, Franz Demmel pour son aide généreuse sur IN3 et Dieter Richter pour son soutien permanent.

\section{Références}

[1] M. Rheinstädter et al., Physica B 350 (2004) 136.

[2] M. Rheinstädter et al., Phys. Rev. Lett. 93 (2004) 108107.

[3] R. Lipowsky and E. Sackmann, editors, Structure and Dynamics of Membranes, Handbook of Biological Physics Vol. 1 (Elsevier, North-Holland, Amsterdam, 1995). 
[4] H. Heller, M. Schaefer and K. Schulten, J. Phys. Chem. 97 (1993) 8343.

[5] W. Pfeiffer et al., Europhys. Lett. 8 (1989) 201.

[6] T. Bayerl, Curr Opin Colloid Interface Sci. 5 (2000) 232.

[7] S. Paula et al., Biophys. J. 70 (1996) 339.

[8] C. Münster et al., Europhys. Lett. 46 (1999) 486.

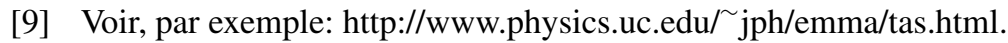

[10] F. Chen, W. Hung and H. Huang, Phys. Rev. Lett. 79 (1997) 4026.

[11] J. Nagle et al., Phys. Rev. E 58 (1998) 7769.

[12] H.I. Petrache et al., Phys. Rev. E 57 (1998) 7014.

[13] C. Liao, S. Chen and F. Sette, Phys. Rev. Lett. 86 (2001) 740.

[14] S. Chen et al., Phys. Rev. Lett. 86 (2001) 740.

[15] T. Weiss et al., Biophys. J. 84 (2003) 3767.

[16] M. Tarek et al., Phys. Rev. Lett. 87 (2001) 238101.

[17] I. de Schepper et al., Phys. Rev. Lett. 50 (1983) 974.

[18] A. van Well et al., Phys. Rev. A 31 (1985) 3391.

[19] A. van Well and L. de Graaf, Phys. Rev. A 32 (1985) 2396.

[20] H. Glyde, Excitations in Liquid and Solid Helium, Oxford Series on Neutron Scattering in Condensed Matter Vol. 9 (Clarendon Press, Oxford, 1994).

[21] P. Mason et al., Phys. Rev. E 63 (2001) 030902(R). 